\title{
Cdc42-dependent leading edge coordination is essential for interstitial dendritic cell migration
}

\author{
Tim Lämmermann, ${ }^{1}$ Jörg Renkawitz, ${ }^{1}$ Xunwei Wu, ${ }^{2}$ Karin Hirsch, ${ }^{1}$ Cord Brakebusch, ${ }^{2}$ and Michael Sixt ${ }^{1}$ \\ ${ }^{1}$ Hofschneider Group Leukocyte Migration, Department of Molecular Medicine, Max Planck Institute of Biochemistry, Martinsried, Germany; and 2Biotech \\ Research and Innovation Centre (BRIC), University of Copenhagen, Copenhagen, Denmark
}

\begin{abstract}
Mature dendritic cells (DCs) moving from the skin to the lymph node are a prototypic example of rapidly migrating amoeboid leukocytes. Interstitial DC migration is directionally guided by chemokines, but independent of specific adhesive interactions with the tissue as well as pericellular proteolysis. Instead, the protrusive flow of the actin cytoskeleton directly drives a basal mode of locomotion that is occasionally supported by actomyosin contractions at the trailing edge to propel
\end{abstract}

the cell's rigid nucleus. We here delete the small GTPase Cdc42 in DCs and find that actin flow and actomyosin contraction are still initiated in response to chemotactic cues. Accordingly, the cells are able to polarize and form protrusions. However, in the absence of Cdc42 the protrusions are temporally and spatially dysregulated, which leads to impaired leading edge coordination. Although this defect still allows the cells to move on 2-dimensional surfaces, their in vivo mo- tility is completely abrogated. We show that this difference is entirely caused by the geometric complexity of the environment, as multiple competing protrusions lead to instantaneous entanglement within 3-dimensional extracellular matrix scaffolds. This demonstrates that the decisive factor for migrating DCs is not specific interaction with the extracellular environment, but adequate coordination of cytoskeletal flow. (Blood. 2009;113: 5703-5710)

\section{Introduction}

Cells of the hematopoietic lineage are fundamentally different from mesenchymal or epithelial cells. Many of them are not restricted to a specific tissue compartment, but rather circulate through the whole organism to perform surveillance or renewal functions. The difference between resident and mobile cells is reflected by the fact that mesenchymal and epithelial cells are constitutively adherent to specific extracellular substrates, whereas hematopoietic cells can flexibly accommodate their adhesive properties. ${ }^{1}$ Dendritic cells (DCs) are excellent examples for both principles as their function relies on the switch from the sessile to the motile state. ${ }^{2}$ The sessile immature DC samples antigens in peripheral tissues. Endogenous or exogenous danger signals trigger the differentiation into the motile state and at the same time induce the expression of the CC-chemokine receptor 7 (CCR7) that directionally guides the cells through the interstitium of the dermis and via the lymphatic vessels into the T-cell area of the lymph node. ${ }^{3,4}$ In the T-cell area, DCs present the peripherally acquired antigens to naive $T$ cells and upon cognate interaction instruct T-cell differentiation and proliferation. Acquisition of the motile state in DCs is accompanied by a functional deactivation of cell-cell adhesion and integrin receptors that allows the cells to detach from their substrates and move freely through the dermal interstitium. ${ }^{5-7}$ Nonadhesiveness endows the cells with enormous flexibility as they become autonomous from the molecular composition of their environment and can directly follow soluble guidance cues like chemokines. We could recently show that nonadhesive DC migration in 3-dimensional (3D) environments can be driven solely by the protrusive flow of actin polymerization at the cell front, whereas actomyosin contractions of the trailing edge served to propel the nucleus through narrow spaces. ${ }^{7}$ Theoretic modeling confirmed that within confined environments actomyosin dynamics can directly drive cellular locomotion without requiring transmembrane force coupling via adhesion receptors. ${ }^{8}$ The fact that 2-dimensional (2D) surfaces, as often used to study cell migration, cannot support such nonadhesive movement explains that cell biology has largely neglected the underlying mechanisms. The migration of leukocytes is likely to be more simplistic than that of adherent cells, as in 3D environments the sheer initiation and polarization of actin flow together with cortical shape changes likely explain most of its aspects.

The universally expressed small GTPases Rac, RhoA, and Cdc 42 are commonly accepted to be the key switches determining cell shape. ${ }^{9,10}$ One of their main functions is the coordination of the actin cytoskeleton and closely interrelated phenomena such as adhesion and microtubule dynamics. It is firmly established that the Rac family members are essential for actin polymerization and RhoA regulates actomyosin contractility. ${ }^{10}$ Both effects have been demonstrated in DCs: Abolishing Rac activity in mature DCs by genetically deleting the 2 expressed isoforms Rac1 and Rac 2 leads to rounding and concomitant immobility of the cells, ${ }^{11}$ which is in line with completely abrogated actin polymerization dynamics. Blockade of RhoA downstream effectors leaves actin protrusion at the leading edge intact, but affects the trailing edge of mature DCs and neutrophils. Here, actomyosin contraction is required to perform squeezing contractions to propel the cell's rigid nucleus through small pores. ${ }^{7}$

The roles of $\mathrm{Cdc} 42$ are less clear cut and many functions such as filopodia formation appear to be cell type-specific. ${ }^{12}$ The most conserved function of $\mathrm{Cdc} 42$ is the coordination of the polarity
Submitted November 26, 2008; accepted January 23, 2009. Prepublished online as Blood First Edition paper, February 3, 2009; DOI 10.1182/blood-2008-11-191882.

An Inside Blood analysis of this article appears at the front of this issue.

The online version of this article contains a data supplement.
The publication costs of this article were defrayed in part by page charge payment. Therefore, and solely to indicate this fact, this article is hereby marked "advertisement" in accordance with 18 USC section 1734. 
module, and many cell types require $\mathrm{Cdc} 42$ to stabilize and maintain spatial and temporal asymmetries. ${ }^{10,13}$ Morphologically, asymmetries usually manifest through the actin cytoskeleton, leading to diverse phenomena such as axon formation or apical basal orientation of epithelia. ${ }^{14}$ In motile leukocytes, polarity determines the direction of movement, and accordingly loss of chemotactic path finding has been observed upon intervention with Cdc42 in neutrophils, ${ }^{15,16}$ macrophages, ${ }^{17}$ hematopoietic stem cells, ${ }^{18}$ and Drosophila melanogaster hemocytes. ${ }^{19}$

The signaling pathways involved in Cdc42-dependent leukocyte polarization have been studied mainly in neutrophils. Here, a positive feedback loop has been described whereupon $\mathrm{G}$ proteincoupled receptor signaling the kinase PAK1 recruits the guanine nucleotide exchange factor $\alpha$ PIX, which activates $\mathrm{Cdc} 42$ that in turn positively regulates PAK1 activity. ${ }^{20}$ In T cells, Cdc42 is activated by Rap1 and induces the Par polarity complex consisting of Par3, Par6, and PKC $\zeta$. This triggers Tiam1, which connects the Par polarity complex to Rap1 at sites of polarity initiation. ${ }^{21}$ Although Cdc42 has not been deleted in DCs until now, studies using dominant-negative versions of the molecule revealed defects in the endocytotic pathway, ${ }^{22}$ antigen presentation, ${ }^{23}$ podosome formation, ${ }^{24}$ and dendrite formation ${ }^{25}$ of immature DCs.

Using DCs deficient for Cdc42, we here address the question of how coordination of actin flow influences migration. Whereas chemotactic movement of Cdc42-deficient DCs is only moderately impaired in 2D assays, it is completely abolished in the dermal interstitium. We show that the severe phenotype results from a defective coordination of multiple protrusions that leads to entanglement in the 3D fibrillar network. These findings demonstrate that the internal stabilization of polarity becomes increasingly important when the cells have to navigate through geometrically complex environments.

\section{Methods}

\begin{abstract}
Animals
Conditional $\mathrm{Cdc} 42^{\text {flox/flox }}$ mice ${ }^{26}$ were intercrossed with $\mathrm{Mx} 1 \mathrm{Cre}$ transgenic mice ${ }^{27}$ to generate $\mathrm{Cdc} 42^{\text {flox/flox }} \mathrm{Mx} 1 \mathrm{Cre}^{+/-}$animals and kept on a mixed 129SV/C57BL/6 genetic background. Control animals were littermates $\left(\mathrm{Cdc} 42^{\text {flox/flox }} \mathrm{Mx} 1 \mathrm{Cre}^{-/-}\right.$or $\left.\mathrm{Cdc} 42^{\text {flox/+ }} \mathrm{Mx} 1 \mathrm{Cre}^{+/-}\right)$. At an age between 6 and 10 weeks, mice received a single intraperitoneal injection of $250 \mu \mathrm{g}$ Poly (I) Poly (C) $(250 \mu \mathrm{g}$; GE Healthcare, Little Chalfont, United Kingdom), diluted in $0.5 \mathrm{~mL}$ phosphate-buffered saline (PBS). Mice were killed 10 to 14 days after injection. The mice were bred in a conventional animal facility at the Max Planck Institute of Biochemistry and according to the local regulations. Mouse breeding and all experimental procedures were approved by the Regierung von Oberbayern.
\end{abstract}

\section{Dendritic cells}

DCs were generated from flushed bone marrow (BM) suspension as previously described in detail. ${ }^{7,28}$ At days 8 to 10 of culture, $200 \mathrm{ng} / \mathrm{mL}$ LPS (Sigma-Aldrich, Munich, Germany; Escherichia coli 0127:B8) was added for 24 hours, and subsequently the culture was depleted of remaining Gr- $1^{+}$ contaminant cells. For depletion, cells were incubated with biotinylated antibodies against Gr-1 (RB6-8C5; BD Pharmingen, Heidelberg, Germany), followed by anti-biotin-microbead separation according to the manufacturer's protocol (Miltenyi Biotec, Bergisch Gladbach, Germany). The purified DCs were used for migration assays. To determine knockout efficiencies, DC lysates were loaded on $12 \%$ polyacrylamide gels for electrophoresis under reduced conditions and Western blotting. Cdc42 was detected with mouse anti-mouse Cdc42 antibody (clone 44; BD Transduction Laboratories, Heidelberg, Germany), and the loading control was performed with rabbit anti-mouse actin antibody (A2066; Sigma-Aldrich). Bound primary antibodies were detected with anti-mouse or anti-rabbit horseradish peroxidase (HRP; both Bio-Rad, Hercules, CA) as secondary antibodies, followed by chemiluminescence with Western Lightning Reagent Plus (PerkinElmer, Boston, MA). Only DC batches without detectable Cdc42 signal were chosen for experiments. Maturation of DCs was analyzed by flow cytometry using antibodies against the mouse antigens: CD11c (HL3, FITC-conjugated), I-A/I-E (2G9), CD40 (3/23), CD86 (GL1; all PE-conjugated; all from BD Pharmingen), and CCR7 (PE-conjugated; eBiosciences, San Diego, CA) with the corresponding isotype controls. ${ }^{7}$ Flow cytometric analysis was performed with a FACSCalibur and CellQuest Pro Software (BD Biosciences, San Diego, CA).

\section{In vivo migration assay}

Gr-1-depleted wild-type (WT) and Cdc42-deficient DCs were labeled with $3 \mu \mathrm{M}$ cell-permeable Cell Trace Oregon Green 488 or $1 \mu \mathrm{M}$ tetramethylrhodamine (TAMRA; both from Invitrogen, Karlsruhe, Germany), respectively, and vice versa. DCs $\left(10^{6}\right)$ at a 1:1 ratio were suspended in $20 \mu \mathrm{L}$ PBS and injected subcutaneously into the hind footpads of C57BL/6 mice. Forty-eight hours after injection, mice were killed by $\mathrm{CO}_{2}$ suffocation, and popliteal lymph nodes were frozen in cryomatrix (Thermo, Pittsburgh, PA) and sectioned in $12-\mu \mathrm{m}$ slices. To visualize the lymph node compartments, cryosections were methanol-fixed, blocked with $1 \%$ BSA in PBS, and stained with pan-laminin antibody (L9393; Sigma-Aldrich) and secondary antirabbit Cy5 (Dianova, Hamburg, Germany). Three different layers of the T-cell cortex of each lymph node were documented using an Axio Imager upright microscope (Zeiss, Jena, Germany). Quantification of DCs within the T-cell cortex was performed with MetaMorph (Molecular Devices, Ismaning, Germany). Using inverse thresholding, the greyscale images of both fluorescence channels were separately binarized. DCs appeared as dots that were quantified using the morphometric analysis tool. The ratio of migrated knockout DCs was calculated as the number of knockout cells divided by the total number of DCs in the T-cell cortex, with 0.5 as the value where $50 \%$ of knockout cells and $50 \%$ WT cells located in the center of the lymph node, and with 0 as the value where only WT cells located in the T-cell cortex of the lymph node.

\section{Ex vivo migration assay}

Ears of killed C57BL/6 mice were removed and mechanically split into dorsal and ventral halves. During separation, the dermis detached from the cartilage layer that remained on one of the halves. LPS-matured, BMderived, magnetic-activated cell sorting (MACS)-purified, TAMRAlabeled DCs were resuspended in culture medium, added on top of the dermis, and incubated for 120 minutes at $37^{\circ} \mathrm{C}, 5 \% \mathrm{CO}_{2}$. For immunofluorescence stainings, ear sheets were then fixed with $1 \%$ paraformaldehyde, incubated with a rabbit polyclonal anti-LYVE-1 antibody (R\&D Systems, Wiesbaden, Germany), washed with PBS, and stained with A488conjugated anti-rabbit secondary antibody (Invitrogen). Images were obtained with a Leica DMIRE2 confocal microscope (objective HCX PL APO/40×/1.25-0.75 oil; Leica, Wetzlar, Germany). Merged z-stacks were generated with the Leica confocal software. For quantification of DC numbers within lymphatic vessels, z-stacks were 3D reconstructed, before the intersection of grayscale images from both fluorescence channels was determined, leaving only DCs within lymphatic vessels to be quantified by integrated morphometry analysis with MetaMorph.

\section{In vitro migration assays}

Three-dimensional collagen gel chemotaxis assays (Figure 6D) have been described earlier. ${ }^{7}$ PureCol (INAMED, Fremont, CA) in $1 \times$ minimum essential medium eagle (Sigma-Aldrich) and $0.4 \%$ sodium bicarbonate (Sigma-Aldrich) was mixed with cells in RPMI (Invitrogen), $10 \%$ fetal calf serum (FCS; Invitrogen) at a 2:1 ratio, resulting in gels with a collagen concentration of $1.6 \mathrm{mg} / \mathrm{mL}$. Final cell concentrations in the assay were $10^{6}$ $\mathrm{DCs} / \mathrm{mL}$ gel. Collagen-cell mixtures were cast in custom-made migration chambers with a thickness of 0.5 to $1 \mathrm{~mm}$. After 30-minute assembly of the 
collagen fibers at $37^{\circ} \mathrm{C}$, the gels were overlayed with $50 \mu \mathrm{L}$ of $0.6 \mu \mathrm{g} / \mathrm{mL}$ CCL19 (R\&D Systems) diluted in RPMI/10\% FCS

For 3D collagen gel invasion assays (Figure 6A), $2.5 \mu \mathrm{g} / \mathrm{mL}$ CCL19 was added before polymerization of the $1.6 \mathrm{mg} / \mathrm{mL}$ collagen gel in a custom-made migration chamber. DCs $\left(5 \times 10^{5}\right)$ were placed on top of the collagen gel, and the chamber was incubated in an upright position for 8 hours at $37^{\circ} \mathrm{C}, 5 \% \mathrm{CO}_{2}$, before images were taken with an inverted Axiovert 40 (Zeiss).

Under-agarose chemotaxis assays (Figure 3A) have been performed as described elsewhere. ${ }^{29}$ Briefly, 2.5\% UltraPure agarose (Invitrogen) in distilled water was heated and mixed with $56^{\circ} \mathrm{C}$ prewarmed RPMI $/ 20 \%$ FCS and $2 \times$ Hank buffered salt solution (Sigma-Aldrich) at a 1:2:1 ratio, resulting in an agarose concentration of $6.25 \mathrm{mg} / \mathrm{mL}$. Two microliters of warm agarose-medium mixture was cast in $3.5-\mathrm{cm}$ cell-culture dishes (BD Falcon, Franklin Lakes, NJ) and allowed to polymerize at room temperature. A responder hole (for DCs) was punched $1 \mathrm{~mm}$ from an attractor hole (for chemokine) into the agarose. After 30 minutes of equilibration at $37^{\circ} \mathrm{C}$, $5 \% \mathrm{CO}_{2}, 5 \times 10^{5}$ DCs were transferred into the responder hole and $1.2 \mu \mathrm{g} / \mathrm{mL} \mathrm{CCL19}$ was filled into the attractor hole. Time-lapse videomicroscopy was initiated when DCs started to migrate between the cell-culture plastic and the agarose. For under-agarose assays with homogenous chemokine (Figure 2A), $2.5 \mu \mathrm{g} / \mathrm{mL}$ CCL19 was added when the agarosemedium mixture cooled to $37^{\circ} \mathrm{C}$ before casting and polymerization. DC suspension $(1 \mu \mathrm{L})$ was injected beneath the agarose with a fine pipette tip, and time-lapse videomicroscopy recording started immediately.

Low-magnification bright-field movies of 3D collagen chemotaxis assays and all agarose assays were recorded at indicated time intervals using inverted Axiovert 40 (Zeiss) cell-culture microscopes, equipped with custom-built climate chambers $\left(5 \% \mathrm{CO}_{2}, 37^{\circ} \mathrm{C}\right.$, humidified) and PAL cameras (Prosilica, Burnaby, BC) triggered by custom-made software (SVS Vistek, Seefeld, Germany). The objectives used were A-Plan $10 \times / 0.25 \mathrm{Ph} 1$ and LD A-Plan $20 \times / 0.3 \mathrm{Ph} 1$ (both Zeiss). Manual tracking was applied to $3 \mathrm{D}$ and under-agarose chemotaxis assays. DCs were tracked over 3 to 4 hours with ImageJ (National Institutes of Health [NIH], Bethesda, MD) and the Manual Tracking Plugin. Speed and directionality parameters were calculated and visualized as plots by analyzing the acquired data with the Chemotaxis and Migration Tool Plugin.

To visualize the 3D morphology of DCs migrating toward CCL19 in a 3D collagen gel (Figure 7A), DCs were labeled with $3.5 \mu \mathrm{M}$ TAMRA (Invitrogen) and imaged with an inverted Zeiss Observer.Z1 microscope equipped with a Yokogawa spinning disc confocal scanhead, Coolsnap HQ2 camera (implemented by Visitron Systems, Pucheim, Germany) and a Plan-APOCHROMAT 40×/0.95 Korr oil objective (Zeiss). Images were $3 \mathrm{D}$ reconstructed in MetaMorph and processed with the Surpass Tool of the Imaris 4.0.6 software (Bitplane, Zürich, Switzerland).

\section{Actin dynamics and total internal reflection microscopy}

DCs were transfected with lifeact:GFP between days 8 and 10 of culture using the primary mouse T-cell kit and the Amaxa nucleoporator (Amaxa, Cologne, Germany). ${ }^{30}$ Cells were transfected according to the manufacturer's recommendations, and immediately after transfection LPS was added for overnight maturation. The actin network of DCs migrating in an underagarose setting was visualized with an inverted Zeiss Axiovert 200M microscope equipped with a total internal reflection setup, Coolsnap HQ2 camera (both Visitron Systems) and a Plan-FLUAR $100 \times / 1.45$ oil objective (Zeiss). Images were taken every 2 seconds. Image analysis was performed with MetaMorph: protrusion and retraction of leading edge regions were presented as time montage after applying the background flattening tool. Actin dynamics were quantified by kymograph analysis, whereby the actin polymerization rate was calculated as the sum of the retrograde actin flow (as observed in some DCs) and the actin forward protrusion at the leading edge.

\section{Statistics}

After data were confirmed to fulfill the criteria, $t$ tests were performed; otherwise, Mann-Whitney $U$ tests were applied. Analyses were performed with Sigma Stat 2.03 (Systat, San Jose, CA).
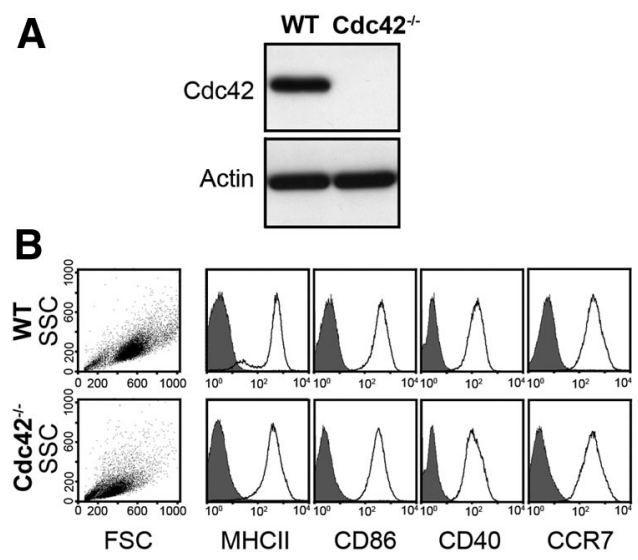

Figure 1. Knockout efficiency and maturation of $\mathrm{Cdc} 42^{-1-}$ dendritic cells. At day 8 of culture, bone marrow (BM)-derived dendritic cells (DCs) were matured with $200 \mathrm{ng} / \mathrm{mL}$ lipopolysaccharide for 24 hours and used for experiments. (A) Cdc42 expression was determined by Western blot analysis of wild-type (WT) and Cdc42 ${ }^{-1-}$ DC lysates. Actin expression was used as loading control. Cdc42 $2^{-1-}$ DCs with no detectable Cdc42 stain were chosen for experiments. (B) Size (dot plots) and maturation (histograms) of WT and $\mathrm{Cdc} 42^{-/-}$DCs were analyzed by flow cytometric analysis. Expression of surface maturation markers on DCs (CD11c-positive cells) was detected with PE-labeled antibodies against MHCII, CD40, CD86, and CCR7 (white open curve) and corresponding isotype controls (gray closed curve). Representative result of independent DC cultures derived from BM of 4 WT and $4 \mathrm{Cdc}_{4} 2^{-1-}$ mice.

\section{Online supplemental material}

Videos S1 through S6 and legends are available on the Blood website (see the Supplemental Materials link at the top of the online article).

\section{Results}

\section{DC differentiation and maturation do not require $\mathrm{Cdc} 42$}

We conditionally deleted $\mathrm{Cdc} 42$ in hematopoietic precursor cells by crossing mice carrying the conditional alleles with transgenics for the inducible Mx1 promoter-driven Cre recombinase. ${ }^{26,27} \mathrm{We}$ generated mature DCs in vitro from BM suspension of mice with induced knockout and obtained cells that were entirely negative for Cdc42 protein as tested by Western blotting (Figure 1A). The cells showed normal DC surface markers including high MHCII and costimulatory molecules and also normal expression levels of chemokine receptor CCR7 (Figure 1B). Cdc42-deficient (Cdc42-/-) DCs had the typical veiled morphology, but were slightly smaller than WT DCs as revealed by the altered scatter profile in flow cytometry (Figure 1B). These findings show that Cdc42 deficiency does not prevent myelopoiesis and DC generation and maturation in vitro. Apart from their smaller size, immature $\mathrm{Cdc} 42^{-1-} \mathrm{DCs}$ were severely impaired in the uptake of fluorescent dextran and also bacterial particles (data not shown), which is in agreement with previous reports that dominant-negative inhibition of $\mathrm{Cdc} 42$ function led to decreased endocytosis and phagocytosis. ${ }^{22,31}$

\section{Symmetry-breaking in response to homogenous chemokine exposure}

To observe the cells upon exposure to polarizing stimuli, we established an assay system that allowed us to follow the morphologic response of DCs by time-lapse video microscopy. As mature DCs are nonadherent, ${ }^{6}$ we chose a setup where the cells are squeezed between a layer of agarose and a coverslip ${ }^{29}$ (Figure 2A). The spatial constraint in this semi-3D setting caused flattening of the cells, leading to a " $2 \mathrm{D}$ projection" that ideally suits to assess 


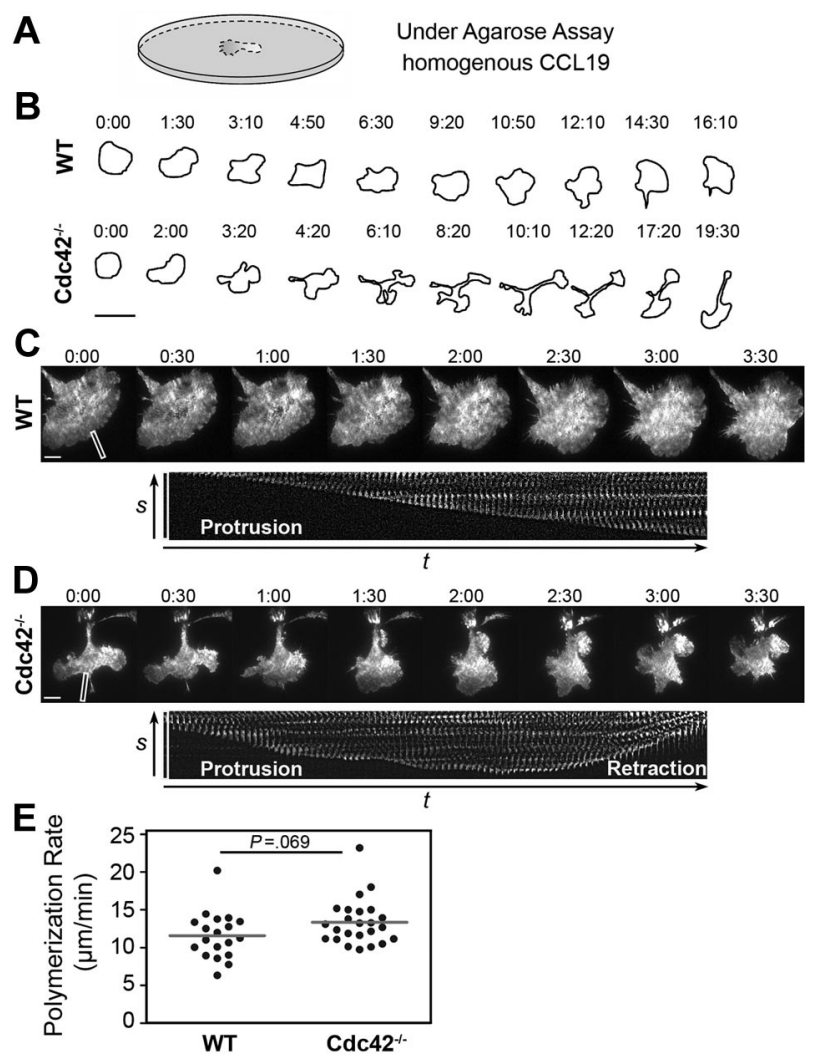

Figure 2. Actin polymerization and coordination at the leading edge of dendritic cells. (A) DCs were injected between a glass coverslip and a layer of agarose containing $2.5 \mu \mathrm{g} / \mathrm{mL}$ CCL19. This experimental setup served for studying the chemokinetic response and actin dynamics of $\mathrm{DCs}$ at $37^{\circ} \mathrm{C}, 5 \% \mathrm{CO}_{2}$ (in panels $\mathrm{B}-\mathrm{E}$ ). (B) Initial radial spreading, symmetry breaking, and subsequent polarization of DCs was recorded by time-lapse videomicroscopy. Morphology outlines of DCs were obtained by morphometric analysis (MetaMorph). A time sequence of a representative WT and Cdc42 ${ }^{-1-}$ DC is shown. Time (minutes:seconds). Scale bar represents $50 \mu \mathrm{m}$. Objective: LD A-Plan 20×/0.30 Ph1 (Zeiss). (C,D) WT (C) and Cdc42-l(D) DCs were transfected with the actin marker lifeact:GFP and actin dynamics observed by TIRF microscopy over time. (Top panels) A time sequence of a representative entire cell is shown. Time (minutes:seconds). Scale bar represents $10 \mu \mathrm{m}$. Objective: Plan-FLUAR $100 \times / 1.45$ oil (Zeiss). (Bottom panels) Leading edge regions indicated by the white boxes were analyzed for phases of protrusion and/or retraction. After background flattening (MetaMorph), a time-lapse montage over 91 seconds (2 seconds/frame) presents the dynamics at the leading edge. (E) Quantification of the actin polymerization rate at the leading edge by kymograph analysis. Cells were derived from 2 independent BM DC cultures (of both WT and Cdc42-1mice). Single cells (dots) were analyzed (line: mean, $t$ test, $P=.069$, WT: $\mathrm{n}=19$, $\mathrm{Cdc}_{2} 2^{-1-}: \mathrm{n}=24$ )

morphologic changes. When WT DCs were exposed to uniform concentrations of the CCR7-binding chemokine CCL19 (Figure $2 \mathrm{~B}$; Video $\mathrm{S} 1$ ), they underwent radial symmetrical expansion until after 2 to 3 minutes the disc shape was reached. This expansion was followed by sudden collapse of radial symmetry and segregation into a leading and a trailing edge, which was accompanied by locomotion. Although some WT cells developed multiple leading edges, the majority polarized along one randomly oriented axis. In Cdc42 $2^{-/-}$DCs, expansion and symmetry breaking occurred equally, demonstrating that CCR7 signaling was intact and that polarization as such was not impaired. However, leading and trailing edge were rarely aligned along one axis. Instead, many cells developed multiple competing leading edges (Figure 2B; Video S1). These observations were in line with previous work demonstrating that $\mathrm{Cdc} 42$ regulates temporal and spatial stability of actin protrusions by either reinforcing one existing protrusion or suppressing the development of new protrusions. ${ }^{32}$ This phenotype motivated us to use $\mathrm{Cdc} 42^{-/-}$DCs as a model system to study the role of actin flow polarization during locomotion.

\section{Locally intact, but globally dysregulated, actin flow in Cdc42 $^{-1-}$ DCs}

Cdc42 is an upstream activator of Rac activity and was further shown to negatively affect RhoA activation in a neutrophil-like cell line. ${ }^{16}$ Both effects might affect the protrusive and contractile aspects of the actin cytoskeleton. To characterize actin dynamics in Cdc42 $2^{-1-}$ DCs, we transfected the cells with lifeact:GFP, a recently described actin-binding peptide, that leaves actin dynamics unaffected and allows dynamic visualization of actin flow within the cells $^{30}$ (Figure 2C,D; Video S2). As an optical setup to image the cortical actin flow, we observed the transfected DCs under agarose with total internal reflection (TIRF) microscopy that excites exclusively the membrane-proximate 100 to $200 \mathrm{~nm}$ of the cell and allows high spatial and temporal resolution while phototoxic effects are minimized. In these assays, polarized WT DCs showed a continuous and expanding sheet of F-actin at the leading edge (Figure 2C), whereas retrograde flow was observed at the lateral aspects of the leading edge (data not shown). In line with our bright-field observations, the morphology of the actin cytoskeleton of $\mathrm{Cdc} 42^{-1-}$ DCs was altered, as many cells developed 2 and sometimes multiple competing leading edges (Figure 2D). Despite this global shape change, local actin flow at the leading edge did not significantly differ from WT cells: kymograph analysis showed that, on short timescale, polymerization rate and accordingly protrusion velocity were unaltered in the absence of Cdc42 ( $t$ test, $P=.069$; Figure 2E). As the persistence time of each protrusion was decreased, protrusion was frequently followed by phases of contraction and retrograde flow (data not shown). These findings show that locally actin protrusion and contraction can be initialized in the absence of $\mathrm{Cdc} 42$, meaning that a principal failure in Rac and RhoA activation is unlikely. It rather occurs that spatial and temporal coordination of actin dynamics is disturbed, leading to multiple competing, instead of one unifying, protrusions.

\section{Chemotactic movement in Cdc42 ${ }^{-I-}$ DCs is moderately impaired in 2D}

We next wanted to test whether this failure to coordinate protrusions affects chemotactic movement. A graded distribution of chemoattractant might preferentially trigger protrusion development toward the high concentration of attractant and, therefore, (partially) counteract the development of competing protrusions that we observed during spontaneous polarization. ${ }^{33}$

We exposed WT cells in the under-agarose assays to diffusion gradients of CCL19 (Figure 3A; Video S3), and observed directionally persistent chemotactic movement toward the chemokine source with single-cell velocities ranging between 5 and $15 \mu \mathrm{m} /$ minutes and high directional persistence (Figure 3B-D). In Cdc42-IDCs, median velocities were only moderately reduced ( $U$ test, $P<.001$ ) and cells still reached $80 \%$ of the WT values (Figure 3C). Although Cdc42 $2^{-1-}$ DCs still showed net movement toward the chemokine, directionality values dropped to $59 \%$ of the WT cells ( $t$ test, $P<.001$; Figure 3B,D). To test whether the decreased directionality was a consequence of impaired coordination of protrusions, we tracked the morphology of the chemotaxing DCs. To exemplify shape changes, we traced the outlines of single cells and displayed cellular morphology alongside instantaneous migration velocities (Figure 3E). Although chemotaxing WT DCs underwent constant shape change, the development of multiple 
A

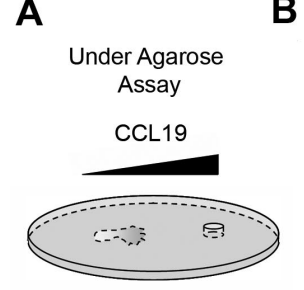

C

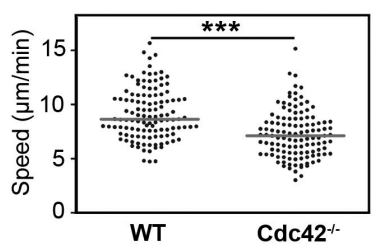

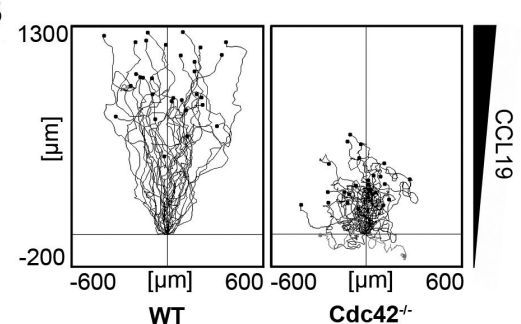

D

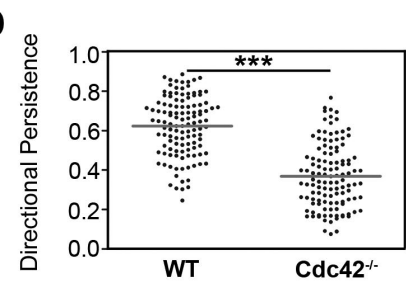

$\mathbf{E}$

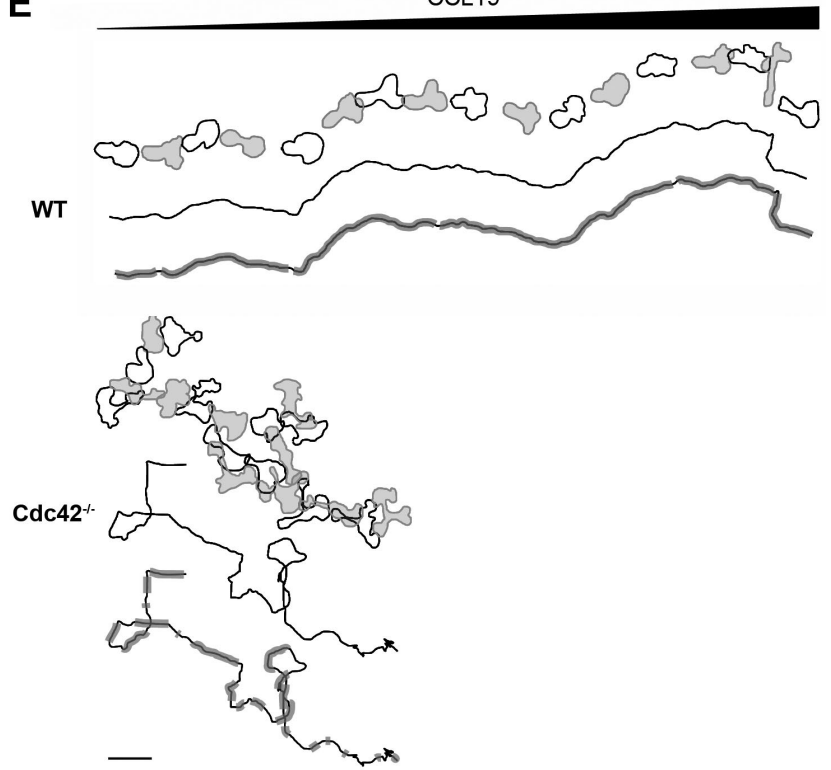

Figure 3. Directed migration of dendritic cells toward a CCL19 gradient in a planar under-agarose assay. (A) Analysis of DC chemotaxis in a planar under-agarose assay. DCs migrate beneath the agarose toward the $1-\mathrm{mm}$ afar attractor hole containing $1.2 \mu \mathrm{g} / \mathrm{mL} \mathrm{CCL} 19$. Directed migration at $37^{\circ} \mathrm{C}, 5 \% \mathrm{CO}_{2}$ was recorded by time-lapse videomicroscopy and analyzed with ImageJ software (in panels B-E). (B) Tracks of single DCs migrating toward a CCL19 gradient over 3 hours ( $n=30$ each). WT indicates wild-type. (C) Comparison of velocity (line: median, $U$ test, $P<.001$ ) and (D) directionality (line: mean, $t$ test, $P<.001$ ) of chemotaxing DCs. Cells were derived from 3 independent BM DC cultures (of both WT and Cdc42 $2^{-1-}$ mice) and applied to individual experiments. Forty single cells (dots) per experiment were tracked (WT: $\mathrm{n}=120$, Cdc42 $\left.{ }^{-l-}: \mathrm{n}=120\right) .{ }^{* \star \star} P<.001$. (E) Correlation of morphology and migration tracks of representative single WT and Cdc42 ${ }^{-1-}$ DCs migrating along a CCL19 gradient. The top track shows outlines of DC morphology over time (WT: 82 minutes, Cdc42 ${ }^{-1-}: 177$ minutes). Morphology outlines of DCs were obtained by morphometric analysis (MetaMorph). The middle track (black line only) represents the migration track, whereas the bottom track indicates migration phases with instantaneous velocities over a threshold speed of $7 \mu \mathrm{m} /$ minutes (gray shaded). Scale bar represents $50 \mu \mathrm{m}$. Objective: A-Plan 10×/0.25 Ph1 (Zeiss).

protrusions was rarely observed. Whenever protrusions branched, retraction of the deviating protrusion occurred almost instantaneously, leading to no significant deceleration of the cell. In addition, Cdc42 $2^{-l-}$ DCs showed phases of high velocities during which they resembled WT cells (Figure 3E, gray-shaded track phases). These phases were frequently interrupted by episodes where the cells displayed multiple competing leading edges, which caused either spinning behavior or complete migratory arrest. Occasionally, multipolar cells even underwent self-fragmentation when multiple protrusions migrated in different directions (data not shown).

Taken together, the graded distribution of chemokine could not compensate for the coordination defect of Cdc42-l- DCs, and the ability of chemotaxing cells to unify the actin flow in one protrusion was impaired. Importantly, this defect led only to slightly diminished migration velocities. Chemotactic migration was still possible in the vertically confined, but horizontally open, 2D setting of the under-agarose assay, albeit directionality was decreased due to the development of competing leading edges.

\section{In vivo migration of $\mathrm{Cdc42}^{-1-} \mathrm{DCs}$ is completely abolished}

Having defined the coordination defect of Cdc42-l- DCs in vitro, we wanted to test how the phenotype manifests in vivo. Bone marrow-derived DCs are well suited for this purpose, as injection into the dermis of mice results in in vivo migration toward the draining lymph node. In an internally controlled experiment, we injected $\mathrm{Cdc}_{2} 2^{-1-}$ DCs together with differentially labeled WT DCs into the footpads of mice and monitored their arrival in draining lymph nodes (Figure 4A). Strikingly, arrival in the T-cell area was completely abolished in the $\mathrm{Cdc} 42^{-1-}$ cells, and we could not detect even trace amounts of these cells either in the sinus area or in the T-cell cortex of the lymph node (Figure 4B,C). This drastic phenotype was somewhat in contrast with our under-agarose assay and raised the question at which level migration was blocked. This could be movement in the dermal interstitium, entry into the lymphatic vessels, or transport toward the node.

To distinguish between a possible block of interstitial migration and impaired entry into the lymphatic vessels, we directly observed the DCs upon entry into the dermal compartment. To this end we used "crawl-in" assays where labeled BM-derived DCs were layered on exposed dermis of explanted murine ear sheets (Figure $5 \mathrm{~A})$. This technique allowed us to monitor dermal movement and lymphatic entry of DCs as the lymphatic vessels were visualized with fluorescent staining against LYVE-1. After 2-hour incubation, WT DCs localized within the lymphatic vessels, whereas $\mathrm{Cdc} 42^{-/-}$ DCs appeared randomly distributed (Figure 5B,C; Video S4). To
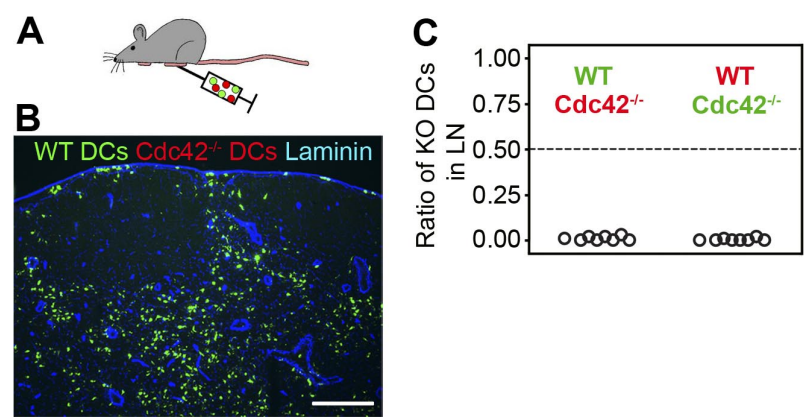

Figure 4. Analysis of in vivo dendritic cell migration from the skin via the lymphatic vessels to the lymph node. (A) Wild-type and Cdc42 $2^{-1-}$ DCs were labeled with TAMRA (red) or Oregon Green 488 (green), and a 1:1 mixture was injected subcutaneously into the hind footpads of C57BL/6 mice and arrival in the popliteal lymph node (LN) was analyzed 48 hours later. (B) Immunofluorescence microscopy (Axio Imager; Zeiss) of $12-\mu \mathrm{m}$-thick LN cryosections. Counterstaining against laminin (Cy5, blue) distinguishes B-cell follicles (B) and T-cell cortex (T). Scale bar represents $200 \mu \mathrm{m}$. Objective: EC Plan-NEOFLUAR 10×/0.3 (Zeiss). (C) Quantification of immunofluorescence analysis. Three different layers of the T-cell cortex of each LN were documented and quantified by morphometric analysis (MetaMorph). Dotted line at $0.5: 50 \%$ of DCs that migrated into the LN are Cdc42 $2^{-1-}$ DCs. Circles indicate single experiments (1 LN). 
A

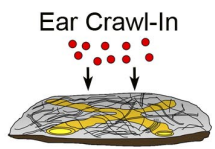

B
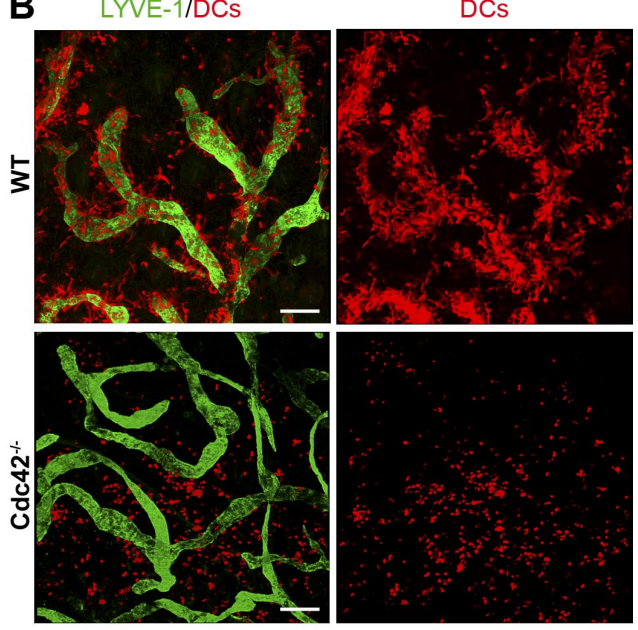

C
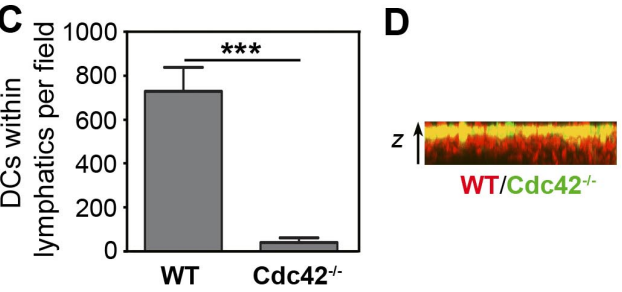

A

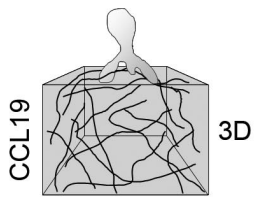

B

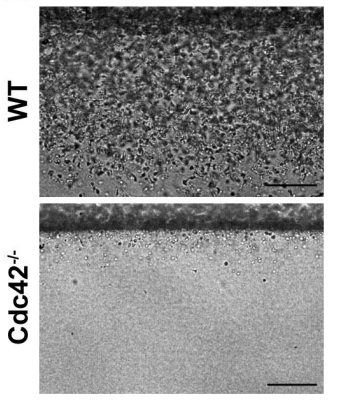

C

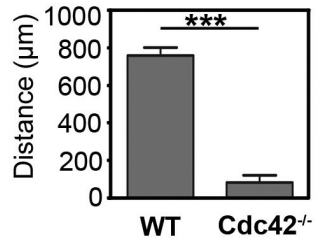

D

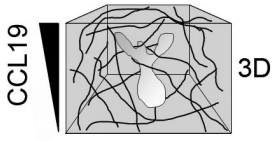

E

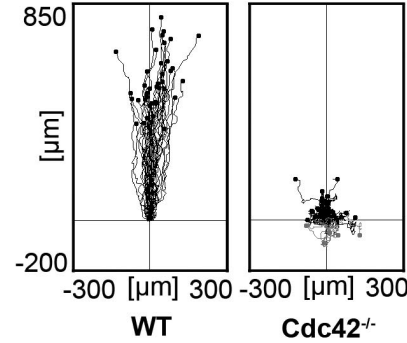

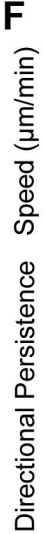

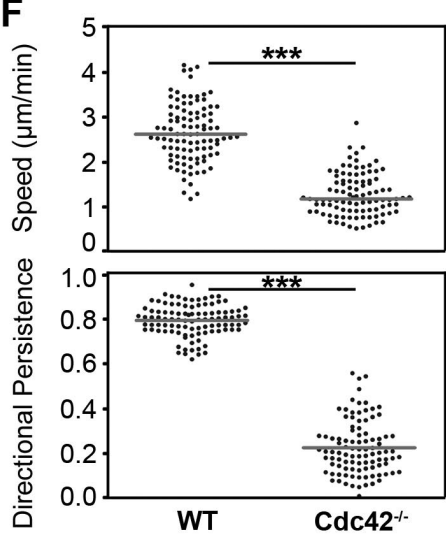

Figure 6. Directed migration of dendritic cells toward a CCL19 gradient in 3D collagen gels. (A) Three-dimensional in vitro setup to study DC tissue invasion (in panels $B, C)$. DCs were layered on top of a polymerized $1.6 \mathrm{mg} / \mathrm{mL}$ collagen gel containing $2.5 \mu \mathrm{g} / \mathrm{mL} \mathrm{CCL} 19$ and incubated at $37^{\circ} \mathrm{C}, 5 \% \mathrm{CO}_{2}$. After 8 hours, DC invasion into the gel was analyzed. (B) Images of WT and $\mathrm{Cdc}^{-1-} 2^{-1} \mathrm{DC}$ invasion were obtained by bright-field microscopy (inverted Axiovert 40; Zeiss). Scale bar represents $200 \mu \mathrm{m}$. Objective: A-Plan 10×/0.25 Ph1 (Zeiss). (C) Cells were derived from 2 independent BM DC cultures (of both WT and Cdc42 ${ }^{-1-}$ mice). Single DC cultures were applied on top of 6 collagen gels and the distance of DC migration into the gel was measured (mean $\pm \mathrm{SD}$, $t$ test, $P<.001$, WT: $\mathrm{n}=12, \mathrm{Cdc}^{-1-}: \mathrm{n}=12$ ). ${ }^{\star * \star} P<.001$. (D) Three-dimensional in vitro setup to study DC interstitial migration. DCs were added to $1.6 \mathrm{mg} / \mathrm{mL}$ collagen, followed by fiber assembly for 30 minutes at $37^{\circ} \mathrm{C}$. Polymerized gels were overlaid with $0.6 \mu \mathrm{g} / \mathrm{mL} \mathrm{CCL} 19$. DC migration at $37^{\circ} \mathrm{C}$, $5 \% \mathrm{CO}_{2}$ was recorded for 4 hours by time-lapse videomicroscopy and analyzed with Image J software (in panels E,F). (E) Tracks of single DCs migrating toward a CCL19 gradient in 3D ( $n=40$ each). (F) Comparison of velocities (line: median, $U$ test, $P<.001$ ) and directionality (line: mean, $t$ test, $P<.001$ ) of DCs chemotaxing in 3D collagen matrices. Cells were derived from 2 independent BM DC cultures (of both WT and $\mathrm{Cdc} 42^{-1-}$ mice) and applied to individual experiments. Fifty single cells (dots) per experiment were tracked (WT: $\left.\mathrm{n}=100, \mathrm{Cdc}^{-/-}: \mathrm{n}=100\right) .{ }^{\star \star \star} P<.001$.

To simulate a complex fibrillar scaffold in the absence of the diverse dermal interstitial matrix molecules, we used 3D collagen gels as migration substrates. We showed previously that collagen gels are excellent substrates for chemotactic DC movement and that, like in vivo, the migration in these assays is independent of adhesion receptors. ${ }^{7}$ We modified the previously described collagen gel chemotaxis assay to test for the invasive potential of DCs and layered WT and Cdc42 $2^{-1-}$ DCs on top of collagen gels that contained CCL19 (Figure 6A). Wild-type cells quickly invaded the gels, whereas $\mathrm{Cdc} 42^{-1-}$ DCs strictly remained on top of the gel and invasion was virtually abolished ( $t$ test, $P<.001$; Figure 6B,C). This indicated that the sheer configuration of the extracellular environment rendered the mutant cells unable to enter.

We next "forced" the cells into the gels by incorporation into the collagen scaffold before exposure to a CCL19 gradient (Figure 6D; Video S5). In line with the invasion assays, WT cells vigorously

Our previous data made it unlikely that the incapability to infiltrate the dermal interstitium was caused by a defect in chemokine sensing. Furthermore, the finding that DCs can migrate in the absence of adhesive interactions with the tissue ${ }^{7}$ argued against Cdc42-I- DCs being unresponsive to other cues of the dermal interstitium, such as extracellular matrix molecules. Hence, we explored the option that not the composition but the geometry of the environment could prevent entry of $\mathrm{Cdc} 42^{-1-}$ DCs. 


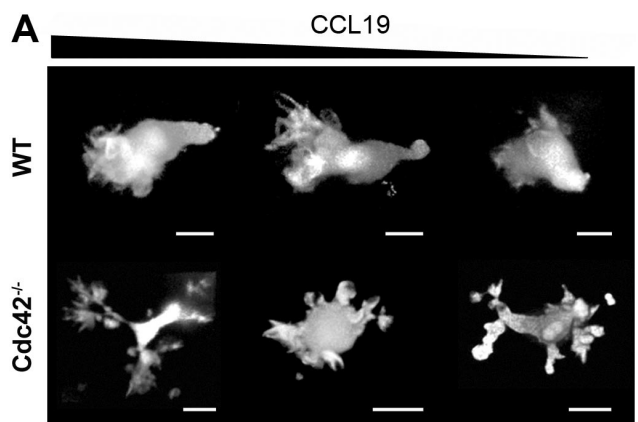

B

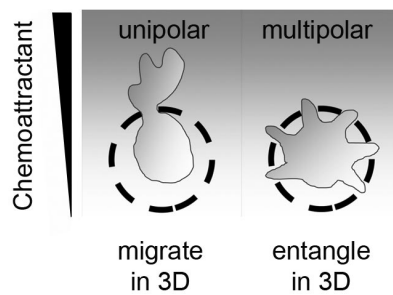

Figure 7. Morphology of migrating dendritic cells in 3D collagen gels and concluding scheme. (A) TAMRA-labeled DCs were added to $1.6 \mathrm{mg} / \mathrm{mL}$ collagen, followed by fiber assembly for 30 minutes at $37^{\circ} \mathrm{C}$. Polymerized gels were overlaid with $0.6 \mu \mathrm{g} / \mathrm{mL}$ CCL19. After 60 minutes, snapshots of DCs migrating at $37^{\circ} \mathrm{C}$, $5 \% \mathrm{CO}_{2}$ in the $3 \mathrm{D}$ collagen gel toward CCL19 were obtained by spinning disc confocal microscopy. DC morphologies result from 3D reconstruction of confocal stacks (MetaMorph) and Surpass processing with Imaris software. Scale bars represent $5 \mu \mathrm{m}$. Objective: Plan-APOCHROMAT 40×/0.95 Korr oil (Zeiss). (B) Concluding scheme about the decisive role of coordinated actin protrusions for leukocyte migration in complex 3D environments.

moved along the chemokine gradient, whereas $\mathrm{Cdc} 42^{-1-}$ DCs had a drastically reduced migration perimeter (Figure 6E). When we visualized the cells in high resolution within the gels, it became apparent that in the absence of $\mathrm{Cdc} 42$ the ability to move long distances was completely abolished as the cells simultaneously sent protrusions in all directions (Figure 7A; Video S6). Such behavior naturally leads to an entanglement of the cell in the dense meshwork. Accordingly, median migration velocities of $\mathrm{Cdc} 42^{-1-}$ DCs in collagen gels dropped to $44 \%$ compared with WT DCs ( $U$ test, $P<.001$ ) and directional persistence was reduced to $25 \%$ ( $t$ test, $P<.001$; Figure $6 \mathrm{~F}$ ).

Taken together, our 3D in vitro results completely resemble the severe in vivo migration defect and demonstrate that a loss of Cdc42-mediated coordination of protrusive actin flow leads to DC trapping in fibrillar networks. We interpret the data as the logical $3 \mathrm{D}$ extension of the moderate phenotype in the $2 \mathrm{D}$ context of the under-agarose assay, which permits unrestricted movement in the horizontal dimension as no physical barriers exist.

\section{Discussion}

Our previous findings showed that interstitial leukocyte migration is nonadhesive and driven mainly by shape changes of the cortical actin network and protrusive flowing of the leading edge. ${ }^{7}$ Hence, it appears especially essential to gain more knowledge how cytoskeletal regulation affects locomotion in hematopoietic cells. We show here that depletion of $\mathrm{Cdc} 42$ in DCs results in a defect of global cytoskeletal coordination, whereas, locally, actin polymerization and actomyosin contraction remains intact. In cells migrating on 2D surfaces, this defect manifests as tumbling and $41 \%$ reduction of directional persistence, although the cells still reach $80 \%$ of the velocities of their WT counterparts. These findings are in line with previous in vitro 2D migration studies that show intact chemokinesis, but impaired chemotaxis in macrophages, ${ }^{17}$ neutrophil granulocytes, ${ }^{15}$ and $\mathrm{T}$ lymphocytes ${ }^{34}$ expressing dominant-negative mutants of $\mathrm{Cdc} 42$. The phenotype also resembles the phenotype of $\mathrm{Cdc} 42^{-1-}$ fibroblastoid cells, which show unimpaired migration speed during wound closure, while directionality is mildly affected. ${ }^{35}$

Surprisingly, the mild manifestation of the phenotype we observed in the $2 \mathrm{D}$ assays is qualitatively different in vivo where migration is almost completely arrested. As DCs migrate in the absence of adhesive interactions, it is unlikely that the different phenotypes result from distinct migration substrates in the $2 \mathrm{D}$ in vitro assays versus the interstitium of the dermis. We rather think that it is entirely due to the spatial configuration of the environment. This conclusion is supported by our finding that we also observed arrested migration in 3D collagen gels, which mimic the geometry of the dermis but not its complex molecular composition. This raises the question why increasing geometric complexity of the environment demands more tightly coordinated cytoskeletal protrusions. DCs and most other quickly migrating leukocytes do not use path-generating mechanisms such as pericellular proteolysis during migration in $3 \mathrm{D}$ environments. ${ }^{36}$ They rather move along the path of least resistance and-if at all-they transiently deform their environment by squeezing contractions of the cell rear. ${ }^{7}$ Hence, for a leukocyte, the interstitial environment can be described as a complex array of fixed pores (scheme, Figure 7B). The migratory process is a series of decisions to traverse the "mostpromising" pore, which mechanistically means to funnel all actin flow into one cellular protrusion. In this scenario, indecisiveness is extremely problematic, as multiple protrusions lead to entanglement. The geometric complexity of the interstitium compared with the unrestricted environment of the $2 \mathrm{D}$ assay explains why the decision problem of $\mathrm{Cdc} 42^{-1-}$ DCs shows different manifestations in vivo and in vitro. In this study, we did not investigate in detail how $\mathrm{Cdc} 42$ regulates the decisive process of actin flow coordination. However, there is a large body of literature involving Cdc42 in molecular feedback loops that promote the stable formation of one leading protrusion while competing protrusions are suppressed. ${ }^{37}$

Interestingly, observations similar to our 2D findings were made in hemocytes of Drosophila embryos after blocking of Cdc42 activity by dominant-negative inhibition: when the chemotaxing cells were tracked in vivo, the inhibited cells reached velocities that were even higher than their WT counterparts but the directional persistence was diminished. ${ }^{19}$ Although these observations in the $3 \mathrm{D}$ environment of a living fly embryo are contradictory with our 3D data at first sight, they are explainable, as animals with an exoskeleton lack an interstitial matrix scaffold. ${ }^{38}$ Like in the unrestricted $2 \mathrm{D}$ assays, entanglement in the nonfibrillar environment appears unlikely, as a cellular or viscous tissue probably allows deformation by the cytoskeletal forces of the hemocyte.

So far, in vivo data on the effects of $\mathrm{Cdc} 42$ depletion in murine leukocytes are limited to a study showing that $\mathrm{Cdc} 42^{-/-}$hematopoietic stem cells are severely impaired in their BM homing and retention capacity. ${ }^{18}$ By investigating DC migration in a physiologic context, we show here that tight regulation of polarized actin is decisive for leukocyte migration in the interstitium. Our findings underline the importance of considering the extracellular context when investigating cell migration-even in cells locomoting independent of the molecular composition of the tissue. We further think that the cellular polarity module might be a promising target 
to influence the interstitial migration of DCs or other types of leukocytes.

\section{Acknowledgments}

We thank Sylvia Cremer for help with statistics and critical reading of the paper and Reinhard Fässler for continuous support.

This work was supported by the German Research Foundation (Bonn, Germany), the Peter Hans Hofschneider Foundation for Experimental Biomedicine (Zürich, Switzerland), and the Max Planck Society (Munich, Germany).

\section{Authorship}

Contribution: T.L. and M.S. designed experiments and wrote the paper; J.R. and K.H. helped with experiments; and X.W. and C.B. generated and contributed $\mathrm{Cdc} 42^{\text {flox/flox }}$ mice.

Conflict-of-interest disclosure: The authors declare no competing financial interests.

Correspondence: Michael Sixt, Max Planck Institute of Biochemistry, Am Klopferspitz 18, 82152 Martinsried, Germany; e-mail: sixt@biochem.mpg.de.

\section{References}

1. Friedl P. Prespecification and plasticity: shifting mechanisms of cell migration. Curr Opin Cell Biol. 2004; $16: 14-23$

2. Randolph GJ, Angeli V, Swartz MA. Dendritic-cell trafficking to lymph nodes through lymphatic vessels. Nat Rev Immunol. 2005;5:617-628.

3. Alvarez D, Vollmann EH, von Andrian UH Mechanisms and consequences of dendritic cell migration. Immunity. 2008;29:325-342.

4. Förster R, Davalos-Misslitz AC, Rot A. CCR7 and its ligands: balancing immunity and tolerance. Nat Rev Immunol. 2008;8:362-371.

5. Schwarzenberger K, Udey MC. Contact allergens and epidermal proinflammatory cytokines modulate Langerhans cell E-cadherin expression in situ. J Invest Dermatol. 1996;106:553-558.

6. van Helden SF, Krooshoop DJ, Broers KC, Raymakers RA, Figdor CG, van Leeuwen FN. A critical role for prostaglandin E2 in podosome dissolution and induction of high-speed migration during dendritic cell maturation. J Immunol. 2006; 177:1567-1574

7. Lämmermann T, Bader BL, Monkley SJ, et al. Rapid leukocyte migration by integrin-independent flowing and squeezing. Nature. 2008;453:51-55.

8. Hawkins RJ, Piel M, Faure-Andre G, et al. Pushing off the walls: a mechanism of cell motility in confinement. Phys Rev Lett. 2009;102:058103.

9. Etienne-Manneville S, Hall A. Rho GTPases in cell biology. Nature. 2002;420:629-635.

10. Heasman SJ, Ridley AJ. Mammalian Rho GTPases: new insights into their functions from in vivo studies. Nat Rev Mol Cell Biol. 2008;9: 690-701.

11. Benvenuti F, Hugues S, Walmsley M, et al. Requirement of Rac1 and Rac2 expression by mature dendritic cells for T cell priming. Science. 2004;305:1150-1153.

12. Wang $L$, Zheng $Y$. Cell type-specific functions of Rho GTPases revealed by gene targeting in mice. Trends Cell Biol. 2007;17:58-64.

13. Etienne-Manneville S. Cdc42: the centre of polarity. J Cell Sci. 2004;117:1291-1300

14. Nelson WJ. Adaptation of core mechanisms to generate cell polarity. Nature. 2003;422:766-774.
15. Srinivasan S, Wang F, Glavas S, et al. Rac and Cdc42 play distinct roles in regulating $\mathrm{PI}(3,4,5) \mathrm{P} 3$ and polarity during neutrophil chemotaxis. J Cell Biol. 2003;160:375-385.

16. Van Keymeulen A, Wong K, Knight ZA, et al. To stabilize neutrophil polarity, PIP3 and Cdc42 aug ment RhoA activity at the back as well as signals at the front. J Cell Biol. 2006; 174:437-445.

17. Allen WE, Zicha D, Ridley AJ, Jones GE. A role for Cdc42 in macrophage chemotaxis. J Cell Biol. 1998:141:1147-1157.

18. Yang L, Wang L, Geiger $\mathrm{H}$, Cancelas JA, Mo J, Zheng Y. Rho GTPase Cdc42 coordinates hematopoietic stem cell quiescence and niche interaction in the bone marrow. Proc Natl Acad Sci U S A. 2007;104:5091-5096.

19. Stramer B, Wood W, Galko MJ, et al. Live imaging of wound inflammation in Drosophila embryos reveals key roles for small GTPases during in vivo cell migration. J Cell Biol. 2005;168:567-573.

20. Li Z, Hannigan M, Mo Z, et al. Directional sensing requires $\mathrm{G}$ beta gamma-mediated PAK1 and PIX alpha-dependent activation of Cdc42. Cell. 2003; 114:215-227.

21. Gérard $A$, Mertens $A E$, van der Kammen RA, Collard JG. The Par polarity complex regulates Rap1- and chemokine-induced T cell polarization. J Cell Biol. 2007;176:863-875.

22. Garrett WS, Chen LM, Kroschewski R, et al. Developmental control of endocytosis in dendritic cells by Cdc42. Cell. 2000;102:325-334.

23. Shurin GV, Tourkova IL, Chatta GS, et al. Smal rho GTPases regulate antigen presentation in dendritic cells. J Immunol. 2005;174:3394-3400.

24. Burns S, Thrasher AJ, Blundell MP, Machesky L, Jones GE. Configuration of human dendritic cell cytoskeleton by Rho GTPases, the WAS protein, and differentiation. Blood. 2001;98:1142-1149.

25. Swetman CA, Leverrier Y, Garg R, et al. Extension, retraction and contraction in the formation of a dendritic cell dendrite: distinct roles for Rho GTPases. Eur J Immunol. 2002;32:2074-2083.

26. Wu X, Quondamatteo F, Lefever T, et al. Cdc42 controls progenitor cell differentiation and betacatenin turnover in skin. Genes Dev. 2006;20: 571-585.
27. Kühn R, Schwenk F, Aguet M, Rajewsky K. Inducible gene targeting in mice. Science. 1995;269: 1427-1429.

28. Lutz MB, Kukutsch N, Ogilvie AL, et al. An advanced culture method for generating large quantities of highly pure dendritic cells from mouse bone marrow. J Immunol Methods. 1999;223: 77-92.

29. Heit B, Kubes P. Measuring chemotaxis and chemokinesis: the under-agarose cell migration assay. Sci STKE. 2003;170:PL5.

30. Riedl J, Crevenna AH, Kessenbrock K, et al. Lifeact: a versatile marker to visualize $\mathrm{F}$-actin. $\mathrm{Na}$ Methods. 2008;5:605-607.

31. Caron E, Hall A. Identification of two distinct mechanisms of phagocytosis controlled by different Rho GTPases. Science. 1998;282:17171721

32. Wedlich-Söldner R, Li R. Spontaneous cell polarization: undermining determinism. Nat Cell Biol. 2003;5:267-270.

33. Charest PG, Firtel RA. Feedback signaling controls leading-edge formation during chemotaxis. Curr Opin Genet Dev. 2006;16:339-347.

34. del Pozo MA, Vicente-Manzanares M, Tejedor R Serrador JM, Sanchez-Madrid F. Rho GTPases control migration and polarization of adhesion molecules and cytoskeletal ERM components in T lymphocytes. Eur J Immunol. 1999;29:36093620.

35. Czuchra A, Wu X, Meyer $\mathrm{H}$, et al. Cdc42 is not essential for filopodium formation, directed migration, cell polarization, and mitosis in fibroblastoid cells. Mol Biol Cell. 2005;16:4473-4484.

36. Wolf K, Müller R, Borgmann S, Bröcker EB, Friedl P. Amoeboid shape change and contact guidance: T-lymphocyte crawling through fibrillar collagen is independent of matrix remodeling by MMPs and other proteases. Blood. 2003;102 3262-3269.

37. Wedlich-Söldner R, Li R. Yeast and fungal morphogenesis from an evolutionary perspective. Semin Cell Dev Biol. 2008;19:224-233.

38. Fessler JH, Fessler LI. Drosophila extracellular matrix. Annu Rev Cell Biol. 1989;5:309-339. 